(c) 2021 Universidad Nacional Autónoma de México, Facultad de Estudios Superiores Zaragoza.

Este es un artículo Open Access bajo la licencia CC BY-NC-ND (http://creativecommons.org/licenses/by-nc-nd/4.0/).

TIP Revista Especializada en Ciencias Químico-Biológicas, 24: 1-11, 2021.

https://doi.org/10.22201/fesz.23958723e.2021.0.293

\title{
Evaluación de quitosano comercial y extractos acuosos de mesocarpio de coco (Cocos nucifera L.) para el control de Rhizopus stolonifer aislado de guanábana (Annona muricata L.): Pruebas in vitro
}

\author{
Héctor Javier Cortés-Rivera ${ }^{1}$, Ramsés Ramón González-Estrada ${ }^{1}$, \\ José Ángel Huerta-Ocampo ${ }^{2}$, Francisco Javier Blancas-Benítez ${ }^{1}$ \\ y Porfirio Gutiérrez-Martínez ${ }^{1 *}$ \\ ${ }^{1}$ Laboratorio Integral de Investigación en Alimentos, Laboratorio de Biotecnología/División de Estudios de \\ Posgrado e Investigación, Tecnológico Nacional de México/I. T. Tepic, Av. Tecnológico \#2595, Col. Lagos del \\ Country, Tepic 63175, Nayarit, México. ${ }^{2}$ Centro de Investigación en Alimentación y Desarrollo, A. C., Lab. de \\ Bioquímica de Proteínas y Glicanos, Hermosillo 83304, Sonora, México. E-mail: *pgutierrez@ittepic.edu.mx
}

\begin{abstract}
RESUMEN
La eficacia antifúngica de los extractos acuosos de mesocarpio de coco (Cocos nucifera L.) (EAC) y el quitosano comercial (QC) aplicados de manera individual y en combinación se evaluó contra Rhizopus stolonifer en términos de crecimiento micelial, esporulación y germinación de las esporas, así como la obtención de los modelos de crecimiento primario en dos temperaturas de almacenamiento $\left(15-25{ }^{\circ} \mathrm{C}\right)$. La aplicación de los EAC al 10\% redujo significativamente el crecimiento micelial de $R$. stolonifer $(58.81 \pm 6.48 \%)$; por otro lado, el QC $(1.5 \%)$ mostró un efecto de control de hasta un $87 \%$, sin embargo, la combinación de los EAC (10\%) con el QC (1.5\%) fue más efectiva al reducir el crecimiento micelial (>93\%). Todos los tratamientos fueron efectivos para disminuir la producción de esporas (>94\%) en comparación con el control (agar). El QC al combinarse con los EAC fue más efectivo inhibiendo la elongación del tubo germinal (>98 \%) comparado con los tratamientos individuales $(<48 \%)$. El modelo modificado de Gompertz mostró un ajuste adecuado para ambos rangos de temperatura ( $>98-99 \%)$ observándose diferencias significativas $(p \leq 0.05)$ entre las variables de velocidad máxima $\left(V_{\text {máx }}\right)$ y periodo de latencia $(\chi)$, no obstante, todos los tratamientos mostraron un efecto fungistático sobre el desarrollo micelial de $R$. stolonifer. La combinación de los EAC y el QC puede ser una alternativa eco-amigable contra la pudrición suave de los frutos de guanábana.
\end{abstract}

Palabras clave: biopolímero, mesocarpio, Cocos nucifera, fitopatógeno, antifúngico.

Evaluation of commercial chitosan and aqueous extracts of coconut shell (Cocos nucifera $L$.) for the control of Rhizopus stolonifer isolated from soursop (Annona muricata L.): In vitro tests

\begin{abstract}
The antifungal effectiveness of aqueous extracts of coconut mesocarp (Cocos nucifera L.) (AEC) and commercial chitosan (CQ) applied individually and in combination was evaluated against Rhizopus stolonifer in terms of mycelial growth, sporulation, spore germination, as well the primary growth models at two storage temperatures $\left(15-25^{\circ} \mathrm{C}\right)$ was obtained. The use of AEC applied individually, significantly reduced the mycelial growth of $R$. stolonifer ( $>45 \%)$; on the other hand, CQ $(1.5 \%)$ showed up to $87 \%$ of control, however, the combination of AEC (10\%) with CQ $(1.5 \%)$ was more effective in reducing mycelial growth $(>93 \%)$. All treatments were effective in inhibiting spore production (>94\%) compared to control (agar). CQ combined with AEC was more effective by inhibiting the germ tube elongation ( $>98 \%)$ compared to the individual treatments $(<48 \%)$. The modified Gompertz model showed an adequate fit for both temperature ranges $(>$ 98-99\%), observing significant differences $(p \leq 0.05)$ between the variables maximum speed $\left(V_{\max }\right)$ and latency period $(\chi)$, however all treatments showed a fungistatic effect on the mycelial development of $R$. stolonifer. The combination of AEC and CQ can be an eco-friendly alternative against soft rot in soursop fruits.
\end{abstract}

Keywords: biopolymer, mesocarp, Cocos nucifera, phytopathogen, antifungal. 


\section{INTRODUCCIÓN}

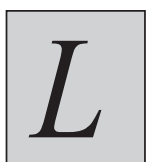

a guanábana (Annona muricata L.) es uno de los cultivos con mayor importancia en algunas regiones tropicales del planeta (Mishra, Ahmad, Kumar \& Sharma, 2013), caracterizada por su pulpa con alto contenido de azúcares y compuestos bioactivos promotores de la salud (Zorofchian et al., 2015). Actualmente, el estado de Nayarit es considerado como el principal productor de guanábana en México, al generar grandes expectativas de crecimiento dentro del sector agrícola (SIAP, 2019), sin embargo, la alta susceptibilidad a infecciones fúngicas ha sido una limitante para su comercialización a nivel nacional e internacional (Berumen-Varela, HernándezOñate, Tiznado-Hernández, 2019). Rhizopus stolonifer es un hongo filamentoso del orden de los Mucorales identificado como el principal agente causal del deterioro fúngico durante el periodo postcosecha del fruto de guanábana dentro del estado de Nayarit (Ramos-Guerrerro et al., 2018). Este tipo de enfermedades han sido relacionadas con una alta dependencia al uso de fungicidas químicos, representando un grave riesgo para la salud humana y el ambiente (Singh \& Sharma, 2018). Debido a lo anterior, se ha buscado el desarrollo de alternativas no contaminantes que permitan el control de estos fitopatógenos y que sean seguras para el consumidor (González-Estrada et al., 2020; Jiménez-Zurita et al., 2017).

En la última década, el quitosano surgió como una opción potencial para la prevención y el tratamiento de algunas de las principales enfermedades fúngicas asociadas a frutos tropicales (Gutiérrez-Martínez et al., 2018). Este polisacárido alcalino con propiedades fungicidas, filmogénicas e inductoras (Romanazzi, Feliziani \& Sivakumar, 2018), es obtenido a partir de la desacetilación parcial de la quitina presente en algunos hongos y exoesqueletos de crustáceos mediante extracción química o enzimática (Betchem, Nana \& Wang, 2019).

Anteriormente, se reportó la aplicación del quitosano grado reactivo con diferentes pesos moleculares $\left(1.74 \times 10^{4}\right.$ $\left.3.07 \times 10^{4} \mathrm{Da}\right)$ para la inhibición del crecimiento micelial de R. stolonifer (30-99\%), destacándose su elevado costo de aplicación y su limitada escalabilidad en campo (HernándezLaurzado et al., 2008; Ramos-Guerrero et al., 2018). Aunque no existen suficientes estudios que comparen la actividad antifúngica entre distintos productos y grados de pureza del polímero (Feliziani et al., 2013; Zhang et al. , 2019), sin embargo, la aplicación del quitosano grado comercial ha demostrado ser una alternativa económica y efectiva para la protección de los productos agrícolas en postcosecha (Pagliarulo et al., 2015; Xing et al., 2018). De igual forma, la biocompatibilidad del quitosano con otros antifúngicos naturales, como podrían ser los compuestos bioactivos presentes en algunos extractos vegetales (Bajic et al., 2019; Siripatrawan \& Harte, 2010), puede generar un mejor espectro de acción antifúngico mediante la mejora de las propiedades funcionales del polímero, así como una mayor estabilidad de los compuestos activos incorporados en su matriz polimérica (Nallamuthu, Devi \& Khanum, 2015; Singh \& Sharma, 2018; Woranuch, Yoksan \& Akashi, 2014; Xie, Hu, Wang \& Zeng, 2014). El aprovechamiento de los subproductos agrícolas como fuente de compuestos bioactivos con potencial antifúngico puede representar una alternativa sustentable para el control de las principales enfermedades fúngicas en frutos tropicales (Fiore \& Cigic, 2019; Ribeiro-da Silva et al., 2014). En este sentido, se han reportado la presencia del ácido clorogénico, flavonoides, alcoholes y otros compuestos orgánicos derivados del metabolismo secundario en plantas y en los extractos acuosos del mesocarpio del coco (Cocos nucifera L.) (Aguilar-Méndez, Campos-Arias, Quiroz-Reyes \& Ronquillo-de Jesús 2019; Cortés-Rivera, González-Estrada \& Blancas-Benítez, 2019a; Dey, Chakraborty \& Mitra, 2005). Estos compuestos bioactivos se han asociado ampliamente con la capacidad de inhibir el desarrollo y la proliferación de algunos patógenos fúngicos importantes como Botrytis cinerea, Penicillium expansum, $P$. digitatum y $R$. stolonifer (Corato, Salimbeni, De Pretis, Avella \& Patruno, 2017; Lagrouh, Dakka \& Bakri, 2017). En un estudio reciente, se reportó la reducción del desarrollo micelial de $P$. italicum en un $80 \%$, así como la disminución en la esporulación en un $98 \%$ y la germinación de esporas en un $99 \%$ mediante la aplicación del extracto acuoso del mesocarpio del coco in vitro (CortésRivera, Blancas-Benítez, Romero-Islas, Gutiérrez-Martínez \& González-Estrada, 2019b). El objetivo del presente trabajo de investigación fue evaluar el efecto antifúngico del quitosano y los extractos acuosos del mesocarpio de C. nucifera aplicados individualmente y en combinación sobre el desarrollo in vitro de $R$. stolonifer.

\section{MATERIALES Y MÉTODOS \\ Materia prima}

El mesocarpio del coco (Cocos nucifera L. var. Criollo) fue adquirido en la ciudad de San Blas, Nayarit, México $\left(21^{\circ} 32^{\prime} 23^{\prime \prime} \mathrm{N} \quad 105^{\circ} 17^{\prime} 08^{\prime \prime} \mathrm{O}\right)$ con productores locales; seleccionando muestras con madurez de consumo y sin presencia de daños durante la cosecha. Las muestras se procesaron en polvo fino de acuerdo con el procedimiento descrito por Cortés-Rivera et al. (2019a). Se usó quitosano comercial $\left(\mathrm{PM}=>3.01 \times 10^{4} \mathrm{Da}, \mathrm{cp}=200,90 \% \mathrm{de}\right.$ desacetilación, América Alimentos S.A. de C.V., Zapopan, Jalisco, México) para los ensayos.

\section{Preparación del extracto acuoso y soluciones de quitosano} Las extracciones acuosas del mesocarpio se llevaron a cabo mediante el método propuesto por Cortés-Rivera et al., (2019a) con algunas modificaciones, $500 \mathrm{mg}$ de mesocarpio en polvo se añadieron en $25 \mathrm{~mL}$ de agua destilada estéril y se mantuvo bajo agitación constante a $300 \mathrm{rpm}$ durante $1 \mathrm{~h}$, el extracto se centrifugó (Hermle Z326 K, EE. UU.) a 6,000 rpm, durante 10 minutos a $4^{\circ} \mathrm{C}$ y se recuperaron los sobrenadantes. 
Los extractos obtenidos se filtraron usando un filtro de jeringa estéril (Unidad de filtro Millex-HN; 0,45 $\mu \mathrm{m}$ de diámetro de poro) y las soluciones se almacenaron en tubos estériles a $4{ }^{\circ} \mathrm{C}$. Las soluciones de QC se prepararon en concentraciones de 0.5 , 1.0 y $1.5 \%(\mathrm{p} / \mathrm{v})$ empleando una solución de vinagre blanco como disolvente y 7\% de ácido acético (Member's Mark, México) al $10 \%(\mathrm{v} / \mathrm{v})$, las soluciones se sometieron a agitación constante durante $24 \mathrm{~h}$, y el pH se ajustó a 5.6 con una solución de $\mathrm{NaOH} 1 \mathrm{~N}$, añadiendo $0.1 \mathrm{~mL}$ de Tween 80 (Sigma-Aldrich, $\mathrm{MO}$, USA) como tensoactivo. Las interacciones consistieron en mezclas detalladas a continuación: QC $0.5 \%+$ EAC $1 \%$, QC 1\% + EAC $5 \%$ y QC $1.5 \%$ + EAC $10 \%$, las soluciones se esterilizaron por separado en autoclave durante (15 min, a $\left.121{ }^{\circ} \mathrm{C}, 15 \mathrm{PSI}\right)$ posteriormente se mezclaron usando un vórtice (Vortex Genie 2, Scientific Industries, EE. UU.) hasta obtener una mezcla homogénea.

\section{Reactivación de Rhizopus stolonifer}

$R$. stolonifer fue aislado de los tejidos de la guanábana infectados e identificado por ensayo molecular mediante la amplificación de las regiones ARNr 18s y 28s (RamosGuerrero et al., 2018), posteriormente fue reactivado agregando $40 \mu \mathrm{L}$ de una suspensión de esporas ( $1 \times 10^{6}$ esporas/ $\mathrm{mL}$ ) en cajas Petri y en un medio enriquecido con guanábana, las cajas fueron incubadas a $25 \pm 2{ }^{\circ} \mathrm{C}$ durante 8 días, el medio de cultivo se preparó mezclando $24 \mathrm{~g}$ de agar bacteriológico $\left(\mathrm{MCD} \mathrm{LAB}^{\mathrm{MR}}\right)$ con $56 \mathrm{~g}$ de cáscara y pulpa de guanábana en madurez de consumo por litro (L) de agua destilada estéril.

\section{Inhibición del crecimiento micelial}

Para la realización de esta prueba se cortaron discos de 10 $\mathrm{mm}$ de diámetro de cultivos de $R$. stolonifer con 4 días de desarrollo y se depositaron en cajas de Petri que contenían los tratamientos con diferentes concentraciones de EAC (1, $5,10 \% \mathrm{v} / \mathrm{v})$, QC $(0.5,1.0$ y $1.5 \% \mathrm{w} / \mathrm{v})$ y las combinaciones (QC $0.5 \%$ + EAC $1 \%$, QC $1 \%+$ EAC $5 \%$ y QC $1.5 \%+$ EAC $10 \%$ ). Las cajas se incubaron a $25 \pm 2{ }^{\circ} \mathrm{C}$ durante 2 días, las placas control (agar) consistieron solo en agar enriquecido con guanábana. Se empleó un control positivo utilizando un fungicida regional para el tratamiento de la guanábana, Tiabendazol a 300 ppm (MERTECT ${ }^{\circledR} 340$ F, ADAMA Ltd., México) y un control negativo empleando vinagre al $10 \%$ ajustado a un $\mathrm{pH}$ de 5.6 con $\mathrm{NaOH} 1 \mathrm{~N}$. Los resultados se reportaron como porcentaje de inhibición del crecimiento micelial en comparación con el control (Agar).

\section{Inhibición de la esporulación}

El efecto de los tratamientos en el proceso de esporulación se evaluó usando las cajas de Petri como el medio en donde se midió el crecimiento del micelio, se añadieron $10 \mathrm{~mL}$ de agua destilada estéril, y se realizó una ruptura por frotación del micelio usando una varilla de vidrio estéril para liberar las esporas. La suspensión se filtró en una gasa estéril y la concentración de esporas se determinó mediante un recuento microscópico utilizando un hemocitómetro (Paul Marienfeld GmbH \& Co., Alemania). Para cada tratamiento, se realizaron 100 observaciones utilizando un microscopio óptico (Motic Instruments Inc. BA300, Canadá). Los resultados se expresaron como número de esporas $/ \mathrm{mL}$.

\section{Germinación de las esporas}

La germinación de las esporas se evaluó depositando 100 $\mu \mathrm{L}$ de una suspensión con las esporas $\left(1 \times 10^{5}\right.$ esporas/ $\mathrm{mL}$ ) en discos de medio de guanábana adicionados con los tratamientos. Las muestras se incubaron a $25 \pm 2{ }^{\circ} \mathrm{C}$ durante $4 \mathrm{~h}$ y se observaron microscópicamente, se analizaron 100 esporas por disco. Las esporas se consideran germinadas cuando el tubo germinal tiene al menos el doble del diámetro de una espora (Ramos-Guerrero et al., 2018). Los resultados se expresaron en porcentajes.

\section{Modelo de crecimiento}

Se modeló el desarrollo radial de $R$. stolonifer sobre los diferentes tratamientos utilizando los rangos de temperatura reportados para el almacenamiento del fruto de guanábana. Se empleó el método descrito por Ochoa-Velasco, NavarroCruz, Vera-López, Palou \& Avila-Sosa (2017) con algunas modificaciones utilizando cajas Petri en un medio de cultivo de guanábana adicionado con las diferentes concentraciones de los EAC, el QC y sus combinaciones. Las cajas Petri fueron inoculadas en el centro con $100 \mu \mathrm{L}$ de la suspensión con esporas $\left(1 \times 10^{5}\right.$ esporas $\left./ \mathrm{mL}\right)$ y se almacenaron a temperatura de almacenamiento $\left(25^{\circ} \mathrm{C}\right)$ y de refrigeración a $15{ }^{\circ} \mathrm{C}$. Se tomaron fotografías del crecimiento radial en periodos de 2 horas y las capturas fueron binarizadas y fraccionadas en imágenes de círculos concéntricos de crecimiento progresivo hasta la colonización completa de la caja Petri. La cinética de crecimiento radial fue ajustada con el Software Find Graph (UNIPIZ Lab, 1.960, EE. UU.) ajustando las curvas a un modelo modificado de Gompertz mediante la siguiente ecuación:

$$
\operatorname{Ln} \frac{N_{t}}{N_{0}}=A \exp \left(-\exp \left(V_{\max } * \frac{e}{A}(\chi-t)+1\right)\right)
$$

Donde:

$N_{t}=$ Crecimiento en el tiempo (t).

$N_{0}=$ Crecimiento en el tiempo (0).

$V_{\text {máx }}=$ Tasa de crecimiento máxima específica.

$A=$ Crecimiento máximo en la fase estacionaria.

$\chi=$ Duración de la fase de latencia.

Los parámetros del modelo se estimaron mediante un análisis de regresión utilizando el software Statistica 10.0 para Windows. El valor del error cuadrático medio (MSE) y el coeficiente de determinación $\left(\mathrm{R}^{2}\right)$ se usaron para determinar la fiabilidad del ajuste del modelo. 


\section{Análisis estadístico}

Se realizó un diseño unifactorial completamente al azar para determinar las diferencias significativas entre los tratamientos utilizados de EAC, QC y las combinaciones. Para las pruebas de inhibición del crecimiento micelial, modelado de crecimiento y esporulación se utilizaron cinco cajas de Petri y cinco discos de agar en las pruebas de germinación de las esporas. Los experimentos se repitieron dos veces de manera independiente. El ANOVA (análisis de varianza) de los datos se aplicó utilizando el software Statistica versión 10.0 para Windows. Las diferencias entre las medias de los datos se compararon mediante la prueba de Fisher LSD, las diferencias $(p \leq 0.05)$ se consideraron significativas.

\section{RESULTADOS Y DISCUSIÓN}

\section{Inhibición del crecimiento micelial}

El crecimiento micelial de $R$. stolonifer fue significativamente diferente $(p \leq 0.05)$ dependiendo del tipo de tratamiento utilizado después de 2 días de incubación (Tabla I). En comparación con el control (agar), todas las concentraciones de los EAC tuvieron un efecto en la reducción del desarrollo micelial de $R$. stolonifer (Figura 1). Se observó un mejor resultado a una concentración del $10 \%$ (58.81 $\pm 6.48 \%$ de inhibición). Estudios previos evaluaron la actividad antifúngica, de los extractos acuosos de diferentes subproductos agrícolas (hojas, tallos y semillas), sobre el crecimiento micelial de $R$. stolonifer, reportando una efectividad in vitro inferior al $10 \%$ de inhibición (BautistaBaños, Hernández-López, Díaz-Pérez \& Cano-Ochoa, 2000; Medda, Hajra, Dey, Bose \& Mondal, 2014), que podría estar relacionada a diferencias en el perfil fitoquímico de los tejidos utilizados para realizar la extracción (Aqil et al., 2010), sugiriéndose además una mayor susceptibilidad de $R$. stolonifer al interactuar con extractos ricos en compuestos fenólicos, flavonoides, terpenos, alcoholes y alcaloides (Bhagwat \& Datar, 2014; Manenji, Mudyiwa, Midzi \& Tsodzo, 2017; Shreya, Hajra, Dey, Bose \& Mondal, 2014; Yang \& Jiang, 2015). En este sentido, se reportó un alto contenido en ácidos hidroxicinámicos, ácido clorogénico, galocatequina y algunos alcoholes de cadena corta relacionados con la actividad antifúngica en los EAC (Aguilar-Méndez et al., 2019; Cortés-Rivera, 2019a; Dey et al., 2005). Aunque el mecanismo de acción de estas moléculas orgánicas no se encuentra totalmente esclarecido, el potencial antifúngico de estos metabolitos secundarios se ha relacionado con la presencia de anillos aromáticos y grupos reactivos capaces de interactuar con las moléculas polares presentes en la membrana fúngica, produciendo su rápida permeabilización y generando alteraciones a nivel intracelular relacionadas con la inactivación de las enzimas del metabolismo basal y disrupción de la membrana mitocondrial (Cerqueira, Barcellos, Machado, Aires \& Dummer, 2015; Martínez et al., 2017; Mohamed, Saleh, Abdel-Farid \& El-Naggar, 2016; Masih, Peter \& Tripathi, 2014; Zaker, 2016).

No se observaron diferencias significativas $(p<0.05)$ entre el control (Agar) y el control negativo (vinagre $10 \%$ ) descartando la actividad antifúngica del vinagre. El QC a concentraciones del $1.5 \%$ mostró mayor inhibición $(87.77$ $\pm 3.10 \%$ ). Anteriormente, fue reportada la capacidad del quitosano para interactuar con las membranas fúngicas de

Tabla I. Porcentaje de inhibición del crecimiento micelial, esporulación y germinación de las esporas de $R$. stolonifer expuesto a diferentes tratamientos por 2 días a $25 \pm 2{ }^{\circ} \mathrm{C}$.

\begin{tabular}{|c|c|c|c|}
\hline Tratamientos & $\begin{array}{c}\text { Inhibición del crecimiento } \\
\text { micelial }(\%)\end{array}$ & $\begin{array}{l}\text { Esporulación } \\
(\text { esporas/mL) }\end{array}$ & $\begin{array}{c}\text { Inhibición de la } \\
\text { germinación }(\%)\end{array}$ \\
\hline Control & $0.0 \pm 0.0^{\mathrm{a}}$ & $2.85 \times 10^{6} \pm 0.53^{\mathrm{a}}$ & $0.0 \pm 0.0^{\mathrm{a}}$ \\
\hline Tiabendazol 300 ppm & $100.0 \pm 0.04^{b}$ & $0.0 \pm 0.0^{\mathrm{b}}$ & $100.0 \pm 0.01^{b}$ \\
\hline EAC $1 \%$ & $48.32 \pm 6.77^{c}$ & $1.4 \times 10^{5} \pm 0.97^{\mathrm{c}}$ & $48.5 \pm 6.53^{c}$ \\
\hline EAC $10 \%$ & $58.81 \pm 6.48^{\mathrm{d}}$ & $0.55 \times 10^{5} \pm 0.6^{\mathrm{d}}$ & $70.5 \pm 2.16^{\mathrm{e}}$ \\
\hline QC $0.5 \%$ & $67.12 \pm 7.45^{\mathrm{e}}$ & $0.45 \times 10^{5} \pm 0.64^{\mathrm{d}}$ & $98.66 \pm 1.75^{f}$ \\
\hline QC $1 \%$ & $83.27 \pm 2.90^{\mathrm{f}}$ & $0.35 \times 10^{5} \pm 0.41^{\mathrm{e}}$ & $99.33 \pm 0.81^{\mathrm{f}}$ \\
\hline QC $1.5 \%$ & $87.77 \pm 3.10^{\mathrm{g}}$ & $0.2 \times 10^{5} \pm 0.25^{\mathrm{f}}$ & $99.66 \pm 0.5^{f}$ \\
\hline
\end{tabular}

Los valores seguidos de la misma letra no son significativamente diferentes con un riesgo del 5\%. Promedio de diez réplicas \pm desviación estándar. 


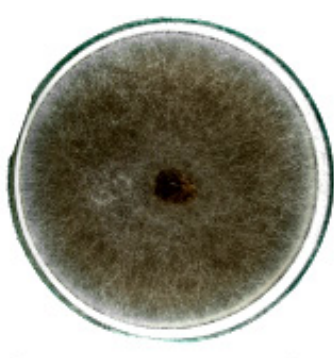

Control

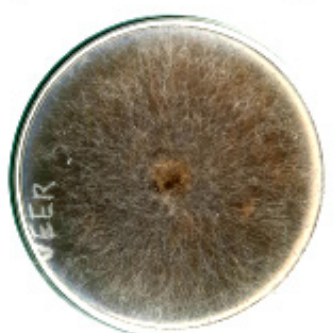

Vinagre $10 \%$

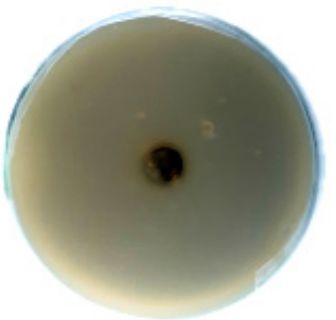

Tiabendazol $300 \mathrm{ppm}$
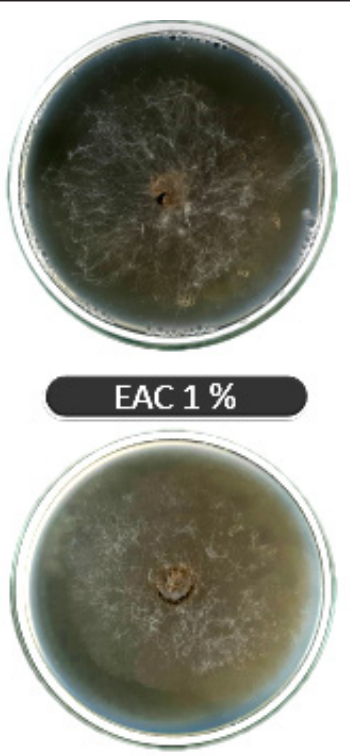

QC $0.5 \%$

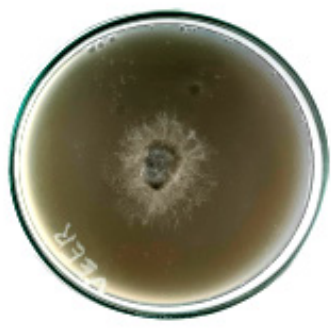

QC $0.5 \%+$ EAC $1 \%$
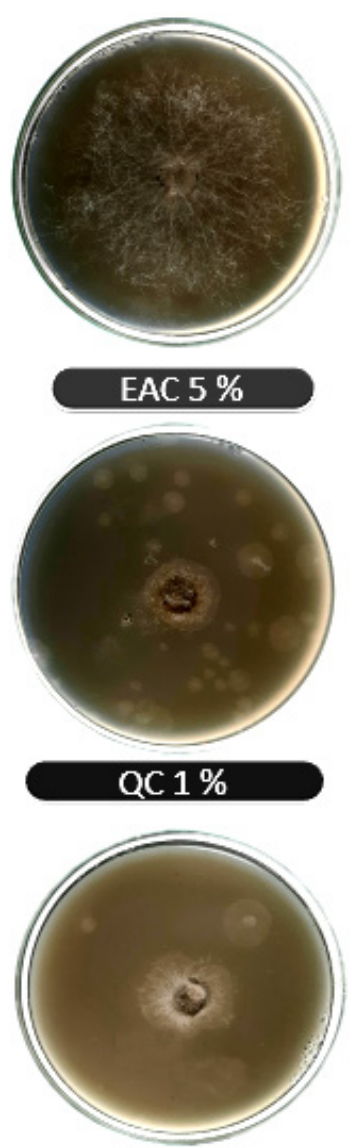

QC $1 \%+$ EAC $5 \%$

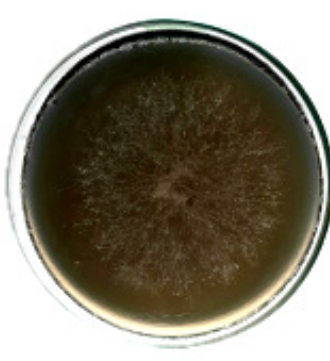

EAC $10 \%$

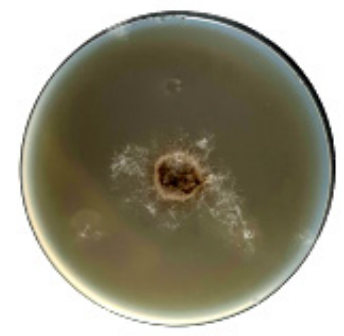

QC $1.5 \%$

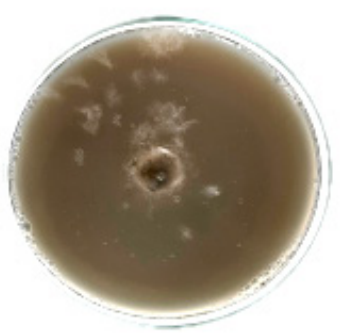

QC $1.5 \%+$ EAC $10 \%$

Figura 1. Crecimiento micelial de $R$. stolonifer expuesto a diferentes tratamientos durante 2 días a $25 \pm 2^{\circ} \mathrm{C}$.

R. stolonifer produciendo permeabilización y pérdida del contenido celular (proteínas y aminoácidos), causando graves alteraciones bioquímicas y morfológicas que dificultan el proceso de infección y desarrollo de las hifas expuestas (García-Rincón et al., 2010; Ghaouth, Arul, Asselin \& Benhamou, 1992; González-Estrada et al., 2020; GutiérrezMartínez et al., 2018).

Previas investigaciones concluyen que la aplicación del quitosano de bajo peso molecular $\left(<2.5 \times 10^{4} \mathrm{Da}\right)$ puede producir un mayor efecto inhibitorio sobre el desarrollo micelial de $R$. stolonifer (hasta 99\%) a partir de concentraciones del 1.5 \% (Hernández-Lauzardo et al., 2008; Ramos-Guerrero et al., 2018).

La efectividad del quitosano con base en el peso molecular se ha relacionado con el número de cargas positivas capaces de interactuar de manera electrostática con la pared del patógeno afianzando su actividad antifúngica (Romanazzi et al., 2018). El quitosano de alto peso molecular tiene una menor presencia de grupos amino disponibles en sus cadenas largas debido a posibles entrelazamientos que explican su menor efectividad in vitro (Feliziani et al., 2013; Niaounakis, 2014). Los EAC y el QC no demostraron ser completamente efectivos en aplicaciones individuales en comparación con el control positivo (100 $\pm 0.0 \%$ de inhibición), sin embargo, la combinación del QC al $1.5 \%$ con los EAC al $10 \%$ resultó ser más efectiva $(94.68 \pm 2.15 \%)$. Se ha reportado el efecto aditivo o sinérgico de las propiedades funcionales del quitosano al ser combinado con diferentes extractos vegetales (Duran et al., 2016; Khalifa, Barakat, El-Mansy \& Soliman, 2017; Muzzalupo, Badolati, Chiappetta, Picci \& Muzzalupo, 2020; Sabaghi, Maghsoudlou, Khomeiri \& Ziaiifar, 2015). Las investigaciones realizadas mencionan una asociación estable del quitosano con algunos compuestos fenólicos mediante interacciones hidrofóbicas, apilamiento $\pi-\pi$, enlaces múltiples de hidrógeno o uniones covalentes entre los grupos reactivos del quitosano y los grupos polares de los compuestos fenólicos (Siripatrawan \& Harte, 2010; Xie et al., 2014; Jiao et al., 2019). 
La aplicación de tratamientos que reduzcan o inhiban el rápido desarrollo micelial de $R$. stolonifer puede ser una estrategia efectiva para el control de las pudriciones blandas en frutos; así como también la afectación de condiciones favorables para la captación de nutrientes se les relaciona con una disminución en la patogenicidad de este hongo (Petrasch et al., 2019).

\section{Esporulación}

La producción de esporas en $R$. stolonifer fue significativamente diferente entre los tratamientos $(p \leq 0.05)$ después de 2 días de incubación. En la Tabla I se muestra la concentración de esporas por tratamiento. No se encontró diferencia significativa $(p>0.05)$ entre el control (agar) y el control negativo (vinagre $10 \%$ ). Los tratamientos de EAC y QC aplicados individualmente demostraron ser efectivos con valores superiores al $90 \%$ de inhibición. En estudios previos se observó que la aplicación de los extractos acuosos ricos en ácidos fenólicos puede tener un mayor efecto sobre los procesos de esporulación en $R$. stolonifer (Bautista-Baños et al., 2000; Bautista-Baños, Hernández-López \& BosquezMolina, 2004). Esto puede deberse a un posible daño estructural en las membranas celulares, así como alteraciones en la actividad enzimática relacionada con los procesos de adaptación y diferenciación de las estructuras formadoras de esporas (Karim et al., 2015; Mohamed et al., 2016).

De igual forma, se observó una reducción similar en la capacidad de producción de las esporas de $R$. stolonifer $(<99 \%)$ al entrar en contacto con el quitosano de alto peso molecular a partir de concentraciones de $0.5 \%$ (GarcíaRincón et al., 2010). Esto puede ser por la capacidad del quitosano para producir desequilibrios en la homeostasis iónica del $\mathrm{Ca}^{2+} \mathrm{y} \mathrm{K}^{+}$en la célula fúngica, produciendo el escape de los nucleótidos, fosfatos, sustratos de reacciones enzimáticas relacionados con la respiración ${ }_{2}$ los procesos de diferenciación, así como a la producción de esporas (BautistaBaños, Ventura-Aguilar, Correa-Pacheco \& Corona-Rangel, 2017; Peña, Sánchez \& Calahorra, 2013).

No se observaron diferencias significativas $(p \leq 0.05)$ entre las combinaciones de los EAC con elQC demostrando ser efectivas respecto al control positivo. La aplicación de tratamientos que permitan la inhibición de los procesos de esporulación en hongos filamentosos, es una estrategia importante en la ruptura del ciclo de infección de los patógenos fúngicos (HeeSoo \& Jae-Hyuk, 2012), en este sentido, la aplicación del QC y los EAC puede ayudar a reducir la virulencia de R. stolonifer sobre frutos susceptibles a su ataque.

\section{Germinación de las esporas}

El porcentaje de germinación de las esporas en $R$. stolonifer fue significativamente diferente entre las concentraciones de los EAC $(p \leq 0.05)$ después de $4 \mathrm{~h}$ de incubación (Tabla I) y la concentración del $10 \%$ de EAC resulto ser más efectiva (70.5 $\pm 2.16 \%$ de inhibición). El proceso de germinación de las esporas es un evento importante para la infección por hongos, los extractos acuosos ricos en compuestos fenólicos como el ácido clorogénico podrían inducir a una mayor inhibición de los mecanismos de germinación, al suprimir la transducción de señales relacionada con la actividad enzimática de las esporas (Karim et al., 2015; Mohamed et al., 2016).

Todos los tratamientos con QC fueron más efectivos para inhibir el proceso de germinación de las esporas $(\geq 98.66$ $\pm 1.75 \%$ de inhibición). Se conoce un patrón entre el peso molecular del quitosano y su efecto sobre los procesos de germinación de las esporas en algunos hongos filamentosos, por consiguiente la aplicación del quitosano de alto peso molecular demostró mejores resultados contra $R$. stolonifer (García-Rincón et al., 2010; Ramos-Guerrero et al., 2018). Esta mayor inhibición se relaciona con la capacidad que tienen los grupos amino $\left(-\mathrm{NH}_{2}\right)^{+}$presentes en las moléculas del quitosano para interactuar con las cargas negativas de la membrana celular de la espora, causando cambios en su permeabilidad y alteraciones a nivel intracelular dificultando el proceso de germinación (González-Estrada et al., 2020). No se observaron diferencias significativas $(p \leq 0.05)$ entre las combinaciones del extracto acuoso con el quitosano.

La germinación de las esporas es considerada como la etapa más importante en la infección y propagación de un patógeno fúngico (Cortés-Rivera et al., 2019b), la inhibición de este proceso puede representar un daño importante sobre los mecanismos y estrategias de proliferación de $R$. stolonifer.

\section{Modelo de crecimiento}

La ecuación modificada de Gompertz mostró un buen ajuste en la descripción del desarrollo micelial de $R$. stolonifer sobre los diferentes tratamientos, observándose un coeficiente de determinación promedio $\left(\mathrm{R}^{2}\right)$ de $0.997 \pm 0.005$ para ambas temperaturas de almacenamiento $\left(15^{\circ} \mathrm{C} / 25^{\circ} \mathrm{C}\right)$. Debido a que $R$. stolonifer presenta un crecimiento lineal a partir de $40 \mu \mathrm{m}$ de longitud del tubo germinal, este modelo matemático puede determinar de manera adecuada las principales variables involucradas en la cinética de desarrollo de este patógeno (Nikkhah, Hashemi, Habibi \& Farhoosh, 2017; Pitt \& Hocking, 2009). El análisis estadístico demostró diferencias significativas $(p \leq 0.05)$ entre las variables de velocidad máxima $\left(V_{\text {máx }}\right)$ y el periodo de latencia $(\chi)$ dependiendo del tipo de tratamiento aplicado (Tabla II). Las concentraciones más altas de los EAC (10\%) y el QC $(1.5 \%)$ produjeron un mayor aumento sobre la fase de latencia $(\lambda)$ del patógeno, así como una mayor reducción en la tasa de crecimiento máximo $\left(V_{\text {máx: }}\right)$; sin embargo, los tratamientos combinados de los EAC con el QC demostraron ser más efectivos al reducir estos parámetros al mínimo en ambas temperaturas de almacenamiento. 
Tabla II. Parámetros modificados del modelo de Gompertz para curvas de crecimiento de $R$. stolonifer expuesto a diferentes tratamientos y temperaturas.

\begin{tabular}{|c|c|c|c|c|c|c|}
\hline \multirow{3}{*}{ Tratamientos } & \multicolumn{6}{|c|}{ Parámetros de Gompertz } \\
\hline & \multicolumn{2}{|c|}{$V_{m a ́ x}(\mathbf{m m} / \mathbf{h})$} & \multicolumn{2}{|c|}{$\chi(\mathbf{h})$} & \multicolumn{2}{|c|}{ Variabilidad ( $\mathbf{R}^{2}$ adj.) } \\
\hline & $15^{\circ} \mathrm{C}$ & $25^{\circ} \mathrm{C}$ & $15^{\circ} \mathrm{C}$ & $25^{\circ} \mathrm{C}$ & $15^{\circ} \mathrm{C}$ & $25^{\circ} \mathrm{C}$ \\
\hline Control & $0.067 \pm 0.02^{\mathrm{a}}$ & $0.266 \pm 0.09^{\mathrm{a}}$ & $1.271 \pm 0.42^{\mathrm{a}}$ & $0.022 \pm 0.01^{\mathrm{a}}$ & 0.991 & 0.994 \\
\hline QC $0.5 \%$ & $0.060 \pm 0.01^{b}$ & $0.230 \pm 0.05^{\mathrm{c}}$ & $2.620 \pm 0.61^{\mathrm{b}}$ & $0.825 \pm 0.06^{\mathrm{b}}$ & 0.997 & 0.986 \\
\hline QC $1 \%$ & $0.057 \pm 0.01^{b}$ & $0.218 \pm 0.04^{\mathrm{d}}$ & $2.818 \pm 0.74^{\mathrm{c}}$ & $1.025 \pm 0.09^{c}$ & 0.996 & 0.981 \\
\hline QC $1.5 \%$ & $0.056 \pm 0.01^{\mathrm{c}}$ & $0.212 \pm 0.02^{\mathrm{d}}$ & $3.002 \pm 0.83^{c}$ & $1.193 \pm 0.14^{\mathrm{c}}$ & 0.997 & 0.984 \\
\hline EAC $1 \%$ & $0.063 \pm 0.02^{b}$ & $0.245 \pm 0.07^{b}$ & $2.069 \pm 0.64^{\mathrm{b}}$ & $0.692 \pm 0.11^{b}$ & 0.995 & 0.991 \\
\hline EAC $5 \%$ & $0.062 \pm 0.02^{\mathrm{c}}$ & $0.239 \pm 0.06^{\mathrm{b}}$ & $2.525 \pm 0.41^{\mathrm{b}}$ & $0.766 \pm 0.21^{\mathrm{b}}$ & 0.996 & 0.990 \\
\hline EAC $10 \%$ & $0.060 \pm 0.01^{\mathrm{c}}$ & $0.228 \pm 0.05^{\mathrm{c}}$ & $2.546 \pm 0.31^{\mathrm{b}}$ & $0.954 \pm 0.18^{b}$ & 0.988 & 0.988 \\
\hline $\mathrm{QC} 0.5 \%+\mathrm{EAC} 1 \%$ & $0.057 \pm 0.02^{\mathrm{c}}$ & $0.226 \pm 0.09^{c}$ & $2.640 \pm 0.38^{\mathrm{c}}$ & $1.401 \pm 0.23^{\mathrm{c}}$ & 0.996 & 0.983 \\
\hline QC $1 \%+$ EAC $5 \%$ & $0.056 \pm 0.01^{\mathrm{d}}$ & $0.217 \pm 0.04^{\mathrm{d}}$ & $2.916 \pm 0.42^{\mathrm{c}}$ & $1.945 \pm 0.28^{\mathrm{d}}$ & 0.996 & 0.986 \\
\hline QC $1.5 \%+$ EAC $10 \%$ & $0.054 \pm 0.01^{\mathrm{e}}$ & $0.202 \pm 0.01^{\mathrm{e}}$ & $3.020 \pm 0.78^{c}$ & $2.272 \pm 0.33^{\mathrm{d}}$ & 0.998 & 0.982 \\
\hline
\end{tabular}

Los valores seguidos de la misma letra no son significativamente diferentes con un riesgo del $5 \%$. Promedio de diez réplicas \pm desviación estándar. $V_{m a ́ x}$ : tasa de crecimiento específica máxima, $\chi$ : duración de la fase de latencia.

Existen pocos informes que describan el efecto de los antifúngicos orgánicos mediante modelos matemáticos, pero se conoce que la aplicación de activos naturales de manera combinada produce un mayor rango de acción sobre $R$. stolonifer al modificar los principales parámetros cinéticos asociados con su desarrollo micelial $\left(\chi, V_{\text {máx }}\right)$ (Ochoa-Velasco et al., 2017). Aunque los mecanismos asociados con la adaptación y el desarrollo de $R$. stolonifer al interactuar con los compuestos fenólicos no se encuentran totalmente esclarecidos, estos podrían implicar afecciones sobre los mecanismos de transporte y/o cambios en los mecanismos celulares que conducen a la incorporación de proteínas y polisacáridos en la zona de extensión de la hifa (Suwanamornler, Sangchote, Chinsirikul, Sane \& Chonhenchob, 2018). De igual forma, se sabe de la capacidad del quitosano para afectar variables como la tasa de crecimiento máxima de $R$. stolonifer, así como prolongar la fase de latencia $(\chi)$, debido a modificaciones en el plasma celular y alteraciones graves en la membrana, que conducen a un aumento en los periodos de adaptación y diferenciación del patógeno (Hernández-Lauzardo et al., 2008; García-Rincón et al., 2010).

La temperatura influyó en mayor medida sobre la dinámica de crecimiento de $R$. stolonifer, sin embargo, todos los tratamientos aplazaron la curva de desarrollo del patógeno (Figura 2), esta adaptación a temperaturas bajas y presencia de compuestos antifúngicos se podría deber a características morfológicas y estructurales como la presencia de hifas cenocíticas carentes de septos, capaces de permitir una translocación rápida de organelos y una mayor absorción de nutrientes, permitiendo el desarrollo de $R$. stolonifer en condiciones de estrés (Pitt \& Hocking, 2009; Sardella,

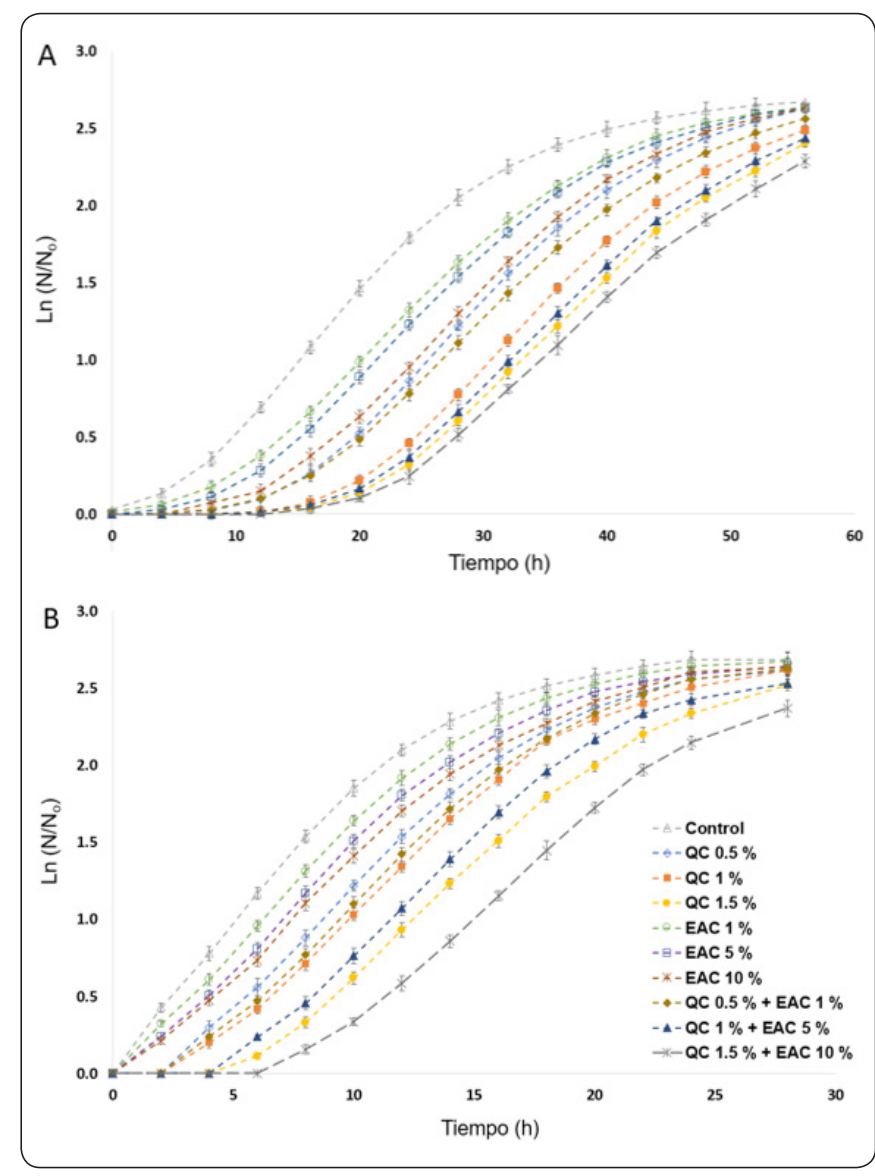

Figura 2. Cinética de crecimiento de $R$. stolonifer. A) Dinámica de crecimiento a $15^{\circ} \mathrm{C}$. B) Dinámica de crecimiento a $25^{\circ} \mathrm{C} . \mathrm{N}=$ Diámetro promedio de la colonia en el tiempo $h, N 0=$ Diámetro de la colonia en el tiempo 0. 
Gatt \& Valdramidis, 2018). Los parámetros de crecimiento determinan el valor de su utilidad al evaluar la actividad antifúngica del QC y los EAC sobre los procesos de infección de $R$. stolonifer en frutos de guanábana.

\section{Conclusiones}

La combinación de los EAC con el QC produce un mayor efecto inhibitorio sobre el crecimiento micelial, esporulación y germinación de las esporas en $R$. stolonifer, así como una mayor modificación en los parámetros cinéticos de crecimiento $\mathrm{V}_{\text {máx }} \mathrm{y} \chi$. Todos los tratamientos producen un efecto fungistático sobre $R$. stolonifer y las combinaciones de los tratamientos muestran un comportamiento sinérgico de manera in vitro, que pueden utilizarse en futuras evaluaciones contra la pudrición suave en la guanábana (A. muricata) y como una alternativa ecológica para la reducción de la patogenicidad y la virulencia de $R$. stolonifer.

\section{Agradecimientos}

Los autores agradecen al Consejo Nacional de Ciencia y Tecnología (CONACYT) por la beca otorgada (731233) a Héctor Javier Cortés Rivera.

\section{REFERENCIAS}

Aguilar-Méndez, M. A., Campos-Arias, M. P., Quiroz-Reyes, C. N. \& Ronquillo-de Jesús M. A. (2019). Fruit peels as sources of bioactive compounds with antioxidant and antimicrobial properties. Rev. FCA UNCUYO, 50, 112. Válido en: http://revistas.uncu.edu.ar/ojs/index.php/ RFCA/article/view/2945/2103.

Aqil, F., Zahin, M., Ahmad, I., Owais, M., Khan, M. S. A., Bansal, S. S. \& Farooq, S. (2010). Antifungal Activity of Medicinal Plant Extracts and Phytocompounds: A Review. In: Ahmad, I. (Ed.) Combating Fungal Infections. (pp. 449-484) Berlin: Springer. DOI: 10.1007/978-3-64212173-9 19

Bajic, M., Rocnik, T., Oberlintner, A., Scognamiglio, F., Novak, U. \& Likozar, B. (2019). Natural plant extracts as active components in chitosan-based films: A comparative study. Food Packaging and Shelf Life, 21, 1-8. DOI: https://doi.org/10.1016/j.fpsl.2019.100365.

Bautista-Baños, S., Hernández-López, M., Díaz-Pérez, J. C. \& Cano-Ochoa, C. F. (2000). Evaluation of the fungicidal properties of plant extracts to reduce Rhizopus stolonifer of "ciruela" fruit (Spondias purpurea L.) during storage. Postharvest Biology and Technology, 20, 99-106. DOI: https://doi.org/10.1016/ S0925-5214(00)00109-5.

Bautista-Baños, S., Hernández-López, M. \& BosquezMolina, E. (2004). Growth inhibition of selected fungi by chitosan and plant extracts. Revista Mexicana de Fitopatología, 22, 178-185. https://www.redalyc.org/ pdf/612/61222204.pdf.
Bautista-Baños, S., Ventura-Aguilar, R. I., Correa-Pacheco, Z. \& Corona-Rangel, M. L. (2017). Chitosan: a versatile antimicrobial polysaccharide for fruit and vegetables in postharvest - a review. Revista Chapingo Serie Horticultura, 23, 103-121. DOI: http://dx.doi. org/10.5154/r.rchsh.2016.11.030.

Betchem, G., Nana A. N. \& Wang, Y. (2019). The application of chitosan in the control of post - harvest diseases: a review. Journal of Plant Diseases and Protection, 126, 495-507. DOI: https://doi.org/10.1007/s41348-01900248-2.

Berumen-Varela, G., Hernández-Oñate, M. A. \& TiznadoHernández, M. E. (2019). Utilization of biotechnological tools in soursop (Annona muricata L.). Scientia Horticulturae, 245, 269-273. DOI: https://doi. org/10.1016/j.scienta.2018.10.028.

Bhagwat, M. K. \& Datar, A. G. (2014). Antifungal activity of herbal extracts against plant pathogenic fungi. Archives of Phytopathology and Plant Protection, 47, 959-965. DOI: https://doi.org/10.1080/03235408.2013.826857

Cerqueira, M. D., Barcellos, H., Machado, P., Aires, J. \& Dummer, D. (2015). Antifungal activity of plant extracts with potential to control plant pathogens in pineapple Diana. Asian Pacific Journal of Tropical Biomedicine, 1, 26-31. DOI: https://doi.org/10.1016/j. apjtb.2015.09.026.

Corato, U., Salimbeni, R., De Pretis, A., Avella, N. \& Patruno, G. (2017). Antifungal activity of crude extracts from brown and red seaweeds by a supercritical carbon dioxide technique against fruit postharvest fungal diseases. Postharvest Biology and Technology, 131, 16-30. DOI: https://doi.org/10.1016/j.postharvbio.2017.04.011.

Cortés-Rivera, H. J., González-Estrada, R. R. \& BlancasBenitez, F. J. (2019a). Extracción e identificación de compuestos bioactivos presentes en residuos de coco (Cocos nucifera) mesocarpio y exocarpio, y su potencial antifúngico. Tecnológico Nacional de México/Instituto Tecnológico de Tepic. pp: 1-65.

Cortés-Rivera, H. J., Blancas-Benítez, F. J., Romero-Islas, L. C., Gutiérrez-Martínez, P. \& González-Estrada, R. R. (2019b). In vitro evaluation of residues of coconut (Cocos nucifera L.) aqueous extracts, against the fungus Penicillium italicum. Emirates Journal of Food and Agriculture, 31, 613-617. DOI: 0.9755/ejfa.2019.v31. i8.1993.

Dey, G., Chakraborty, M. \& Mitra, A. (2005). Profiling C6C3and C6-C1phenolic metabolites in Cocos nucifera. Journal of Plant Physiology, 162, 375-381. DOI: 10.1016/j.jplph.2004.08.006.

Duran, M., Aday, M. S., Zorba, N. N. D., Temizkan, R., Büyükcan, M. B. \& Caner, C. (2016). Potential of antimicrobial active packaging "containing natamycin, nisin, pomegranate and grape seed extract in chitosan coating" to extend shelf life of fresh strawberry. Food and 
Bioproducts Processing, 98, 354-363. DOI: 10.1016/j. fbp.2016.01.007.

Feliziani, E., Romanazzi, G., Margosan, D. A., Mansour, M. F., Smilanick, J. L., Gu, S., Gohil, H. L. \& Rubio-Ames, Z. (2013). Preharvest fungicide, potassium sorbate, or chitosan use on quality and storage decay of table grapes. Plant Disease, 97, 307-314. DOI: http://dx.doi. org/10.1094/ PDIS-12-11-1043-RE.

Fiore, A. \& Cigic, V. V. (2019). Bioactive compounds from food byproducts. Journal of Food Quality, 2019, 1-2. DOI: https://doi.org/10.1155/2019/6213792.

García-Rincón, J., Vega-Pérez, J., Guerra-Sánchez, M. G., Hernández-Lauzardo, A. N., Peña-Díaz, A. \& Velázquez del Valle, M. G. (2010). Effect of chitosan on growth and plasma membrane properties of Rhizopus stolonifer (Ehrenb.: Fr.) Vuill. Pesticide Biochemistry and Physiology, 97, 275-278. DOI: 10.1016/j. pestbp.2010.03.008.

Ghaouth, A. E., Arul, J., Asselin, A. \& Benhamou, N. (1992). Antifungal activity of chitosan on post-harvest pathogens: induction of morphological and cytological alterations in Rhizopus stolonifer. Mycological Research, 96, 769-779. DOI: https://doi.org/10.1016/ S0953-7562(09)80447-4.

González-Estrada, R. R., Vega-Arreguín, J., RoblesVillanueva, B. A., Velázquez-Estrada, R. M., RamosGuerrero, A. \& Gutiérrez-Martínez, P. (2020). Evaluación in vitro de productos químicos no convencionales para el control de Penicillium citrinum. Polibotánica, 49, 161172. DOI: $10.18387 /$ polibotanica.49.11.

Gutiérrez-Martinez, P., Ledezma-Morales, A., Romero-Islas, L. C., Ramos-Guerrero, A., Romero-Islas, J., RodríguezPereida, C., Casas-Junco, P., Coronado-Partida, L. \& González-Estrada, R. R. (2018). Antifungal activity of chitosan against postharvest fungi of tropical and subtropical fruits. In: Dongre, R. S. (Ed.) Chitin-Chitosan - Myriad Functionalities in Science and Technology. (pp. 311-322) London: IntechOpen. DOI: 10.5772/ intechopen.76095.

Hee-Soo, P. \& Jae-Hyuk, Y. (2012) Genetic control of asexual sporulation in filamentous fungi. Current Opinion in Microbiology. 15, 669-677. DOI: https://doi. org/10.1016/j.mib.2012.09.006.

Hernández-Lauzardo, A. N., Bautista-Baños, S., Velázquezdel Valle, M. G., Méndez-Montealvo, M. G., SánchezRivera, M. M. \& Bello-Pérez, L. A. (2008). Antifungal effects of chitosan with different molecular weights on in vitro development of Rhizopus stolonifer (Ehrenb.:Fr.) Vuill. Carbohydrate Polymers, 73, 541-547. DOI: 10.1016/j.carbpol.2007.12.020.

Jiao, W., Chu, S., Li, X., Cao, J., Fan, X. \& Jiang, W. (2019). Preparation of a chitosan-chlorogenic acid conjugate and its application as edible coating in postharvest preservation of peach fruit. Postharvest Biology and
Technology, 154, 129-136. DOI: https://doi.org/10.1016/j. postharvbio.2019.05.003.

Jiménez-Zurita, J. O., Balois-Morales, R., Alia-Tejacal, I., Sánchez-Herrera, L. M., Jiménez-Ruiz, E. I., Bello-Lara, J. E., García-Paredes, J. D. \& Juárez-López, P. (2017). Cold Storage of Two Selections of Soursop (Annona muricata L.) in Nayarit, Mexico. Journal of Food Quality, 1-9. DOI: https://doi.org/10.1155/2017/4517469.

Karim, H., Boubaker, H., Askarne, L., Talibi, I., Msanda, F., Saadi, B., Ait, A. \& Aoumar, B. (2015). Antifungal properties of organic extracts of eight Cistus L. species against postharvest citrus sour rot. Letters in Applied Microbiology, 62, 16-22. DOI: https://doi.org/10.1016/j. micpath.2017.01.041.

Khalifa, I., Barakat, H., El-Mansy, H. A. \& Soliman, S. A. (2017). Preserving apple (Malus domestica var. Anna) fruit bioactive substances using olive wastes extractchitosan film coating. Information Processing in Agriculture. 4, 90-99. DOI: 10.1016/j. inpa.2016.11.001.

Lagrouh, F., Dakka, N. \& Bakri, Y. (2017). The antifungal activity of Moroccan plants and the mechanism of action of secondary metabolites from plants. Journal de Mycologie Medicale, 27, 303-311. DOI: 10.1016/j. mycmed.2017.04.008.

Manenji, B. T., Mudyiwa, R. M., Midzi, J. \& Tsodzo, A. (2017). Antifungal effects of botanical leaf extracts of Lantana camara, Moringa oleifera, and Tagetes minuta on Rhizopus stolonifer in vitro. Journal of Agruculture and Ecology Research International, 11,1-8.DOI: https:// doi.org/10.9734/JAERI/2017/28371.

Martínez, G., Regente, M., Jacobi, S., Del Rio, M., Pinedo, M. \& Del Canal, L. (2017). Chlorogenic acid is a fungicide active against phytopathogenic fungi. Pesticide Biochemistry and Physiology, 140, 30-35. DOI: https:// doi.org/10.1016/j.pestbp.2017.05.012.

Masih, H., Peter, J. K. \& Tripathi, P. A. (2014). Comparative evaluation of antifungal activity of medicinal plant extracts and chemical fungicides against four plant pathogens. International Journal of Current Microbiology and Applied Sciences, 3, 97-109. Disponible en: https:// www.ijcmas.com/vol-3-5/Harison\%20Masih, $\% 20$ et\%20 al.pdf.

Medda, S., Hajra, A., Dey, U., Bose, P. \& Mondal, N. K. (2014). Biosynthesis of silver nanoparticles from Aloe vera leaf extract and antifungal Biosynthesis of silver nanoparticles from Aloe vera leaf extract and antifungal activity against Rhizopus sp . and Aspergillus sp. Applied Nanoscience, 5, 875-880. DOI: https://doi.org/10.1007/ s13204-014-0387-1.

Mishra, S., Ahmad, S., Kumar, N. \& Sharma, B.K. (2013). Annona muricata (the cancer killer): A review. Global Journal Pharmacy, 2, 1613-1618. Válido en: https:// www.yumpu.com/en/document/view/14436104/annonamuricata-the-cancer-killer-a-review. 
Mohamed, M. S. M., Saleh, A. M., Abdel-Farid, I. \& El-Naggar, S. A. (2016). Growth, hydrolases and ultrastructure of Fusarium oxysporum as affected by phenolic rich extracts from several xerophytic plants. Pesticide Biochemistry and Physiology, 141, 57-64. DOI: 10.1016/j.pestbp.2016.11.007.

Muzzalupo, I., Badolati, G., Chiappetta, A., Picci, N. \& Muzzalupo, R. (2020). In vitro Antifungal Activity of Olive (Olea europaea) Leaf Extracts Loaded in Chitosan Nanoparticles. Frontiers in Bioengineering and Biotechnology, 8, 1-10. DOI: 10.3389/ fbioe.2020.00151.

Nallamuthu, I., Devi, A. \& Khanum, F. (2015). Chlorogenic acid loaded chitosan nanoparticles with sustained release property, retained antioxidant activity and enhanced bioavailability. Asian Journal of Pharmaceutical Sciences, 10, 203-211. DOI: http:// dx.doi.org/10.1016/j. ajps.2014.09.005.

Niaounakis, M. 2014. Manufacture of biocomposites, En Niaounakis, M. (Ed.). Biopolymers: Processing and Products. (pp. 411-431) New York: Elsevier. DOI: http:// dx.doi.org/10.1016/B978-0-323-26698-7.00012-X.

Nikkhah, M. Hashemi, M., Habibi, M. \& Farhoosh, R. (2017). Synergistic effects of some essential oils against fungal spoilage on pear fruit. International Journal of Food Microbiology, 257, 285-294. DOI: 10.1016/j. ijfoodmicro.2017.06.021.

Ochoa-Velasco, C. E., Navarro-Cruz, A., Vera-López, O., Palou, E. \& Avila-Sosa. R. (2017). Growth modeling to control (in vitro) Fusarium verticillioides and Rhizopus stolonifer with thymol and carvacrol. Revista Argentina de Microbiología, 50, 70-74. DOI: http://dx.doi. org/10.1016/j.ram.2016.11.010 0.

Pagliarulo, C., Sansone, F., Moccia, S., Russo, G. L., Aquino, R. P., Salvatore, P., Stasio, M. D. \& Volpe, M. G. (2015). Preservation of strawberries with an antifungal edible coating using peony extracts in chitosan. Food and Bioprocess Technology, 9, 1951-1960. DOI: 10.1007/ s11947-016-1779-x.

Peña, A., Sánchez, N. S. \& Calahorra, M. (2013). Effects of chitosan on Candida albicans: conditions for its antifungal activity. BioMed Research International, 2013, 1-15. DOI: 10.1155/2013/527549.

Petrasch, S., Silva, C. J., Mesquida-Pesci, S. D., Gallegos, K., van den-Abeele, C., Papin V., Fernandez-Acero, F. J., Knapp S. J. \& Blanco-Ulate, B. (2019). Infection strategies deployed by Botrytis cinerea, Fusarium acuminatum, and Rhizopus stolonifer as a function of tomato fruit ripening stage. Frontiers in Plant Science, 10, 1-24. DOI: 10.3389/ fpls.2019.00223.

Pitt, J. I. \& Hocking, A. D. (2009). Zygomycetes. En Pitt, J. I. (Ed.) Fungi and Food Spoilage. (pp.145-168). Boston: Springer. DOI: https://doi.org/10.1007/978-0-38792207-2 6.
Ramos-Guerrero, A., González-Estrada, R. R., Hanako-Rosas, G., Bautista-Baños, S., Acevedo-Hernández, G., TiznadoHernández, M. E. \& Gutiérrez-Martínez, P. (2018). Use of inductors in the control of Colletotrichum gloeosporioides and Rhizopus stolonifer isolated from soursop fruits: in vitro tests. Food Science and Biotechnology, 27, 755763. https://doi.org/10.1007/s10068-018-0305-5.

Ribeiro-da Silva, L. M., Teixeira-de Figueiredo, E. A., Silva-Ricardo, N. M. P., Pinto-Vieira, I. G., Wilanede Figueiredo, R., Montenegro-Brasil, I. \& Gomez, C. L. (2014). Quantification of bioactive compounds in pulps and by-products of tropical fruits from Brazil. Food Chemistry, 143, 398-404. DOI: 10.1016/j. foodchem.2013.08.001.

Romanazzi, G., Feliziani, E. \& Sivakumar, D. (2018). Chitosan, a biopolymer with triple action on postharvest decay of fruit and vegetables: Eliciting, antimicrobial and film-forming properties. Frontiers in Microbiology, 9, 27 45. DOI: $10.3389 /$ fmicb.2018.02745.

Sabaghi, M., Maghsoudlou, Y., Khomeiri, M. \& Ziaiifar, A.M. (2015). Active edible coating from chitosan incorporating green tea extract as an antioxidant and antifungal on fresh walnut kernel. Postharvest Biology and Technology, 110, 224-228. DOI: 10.1016/j. postharvbio.2015.08.025.

Sardella, D., Gatt, R. \& Valdramidis, V. P. (2018). Modelling the growth of pear postharvest fungal isolates at different temperatures. Food Microbiology, 76, 450-456. https:// doi.org/10.1016/j.fm.2018.07.010

Shreya, M., Hajra, A., Dey, U., Bose, P. \& Mondal, N. (2014). Biosynthesis of silver nanoparticles from Aloe vera leaf extract and antifungal activity against Rhizopus sp. and Aspergillus sp. Applied Nanoscience, 5, 875-880. DOI: 10.1007/s13204-014-0387-1.

SIAP. (2019). Cierre de la producción agrícola por estado. Anuario Estadístico de la Producción Agrícola de guanábana en México. Disponible en: https://nube.siap. gob.mx/cierreagricola/. Consultado: 29 de noviembre del 2020 .

Singh, D. \& Sharma, R. R. (2018). Postharvest diseases of fruits and vegetables and their management. En Mohammed, W. S. (Ed.). Postharvest Disinfection of Fruits and Vegetables. Academic Press, (pp. 1-52) New York: Elsevier. DOI:10.31018/jans.v11i2.2053.

Siripatrawan, U. \& Harte, B. R. (2010). Physical properties and antioxidant activity of an active film from chitosan incorporated with green tea extract. Food hydrocolloids, 24, 770-775. https://doi.org/10.1016/j. foodhyd.2010.04.003.

Suwanamornlert, P., Sangchote, S., Chinsirikul, W., Sane, A. \& Chonhenchob, V. (2018). Antifungal activity of plant-derived compounds and their synergism against major postharvest pathogens of longan fruit in vitro. International Journal of Food Microbiology, 257, 285294. https://doi.org/10.1016/j.ijfoodmicro.2018.02.009. 
Woranuch, S., Yoksan, R. \& Akashi, M. (2014). Ferulic acidcoupled chitosan: Thermal stability and utilization as an antioxidant for biodegradable active packaging film. Carbohydrate Polymers, 115, 744-751. DOI: https://doi. org/10.1016/j.ijfoodmicro.2018.02.009.

Xie, M., Hu, B., Wang, Y. \& Zeng, X. (2014). Grafting of gallic acid onto chitosan enhances antioxidant activities. Journal of Agricultural and Food Chemistry, 62, 91289136. DOI: 10.1021/jf503207s.

Xing, K., Li, T. J., Liu, Y. F., Zhang, J., Zhang, Y., Shen, X.Q., Li, X.Y., Miao, X. M., Feng, Z. Z., Peng, X., Li, Z.Y. \& Qin, S. (2018). Antifungal and eliciting properties of chitosan against Ceratocystis fimbriata in sweet potato. Food Chemistry, 268, 188-195. DOI: 10.1016/j. foodchem.2018.06.088.

Yang, X. \& Jiang, X. (2015). Antifungal activity and mechanism of tea polyphenols against Rhizopus stolonifer.
Biotechnology Letters, 37, 1463-1472. doi:10.1007/ s10529-015-1820-6.

Zaker, M. (2016). Natural plant products as eco-friendly fungicides for plant diseases control- a review. The Agriculturists, 14, 134-141. DOI: 10.3329/agric.v14i1.29111.

Zhang, W., Zhao, H., Zhang, J., Sheng, Z., Cao, J. \& Jiang, W. (2019). Different molecular weights chitosan coatings delay the senescence of postharvest nectarine fruit in relation to changes of redox state and respiratory pathway metabolism. Food Chemistry, 289, 160-168. DOI: https:// doi.org/10.1016/j.foodchem.2019.03.047.

Zorofchian, M. S., Fadaeinasab, M., Nikzad, S., Mohan, G., Mohd, A. H. \& Kadir, H. A. (2015). Annona muricata (Annonaceae): A review of its traditional uses, isolated acetogenins and biological Activities. Internatioal Journal of Molecular Sciences, 16, 15625-15658. DOI:10.3390/ ijms160715625. 